\title{
Sense of Direction: General Factor Saturation and Associations with the Big-Five Traits
}

\author{
David M. Condon ${ }^{1, \star}$, Joshua Wilt ${ }^{2}$, Cheryl Ann Cohen ${ }^{1}$, William Revelle ${ }^{1}$, \\ Mary Hegarty ${ }^{3}$, David H. Uttal ${ }^{1}$
}

\section{Abstract}

The ability to locate and orient ourselves with respect to environmental space is known as sense of direction ("SOD"). While there is considerable evidence for the predictive utility of self-report measures of this psychological construct, relatively little research has investigated the psychometric properties of the self-report scale by which it is most commonly measured - the Santa Barbara Sense of Direction scale (SBSOD, Hegarty, Richardson, Montello, Lovelace, and Subbiah, 2002) - or the broader personality correlates. The present study evaluated the factor structure of the SBSOD following administration to 12,155 individuals and situated it among prominent sources of individual differences, specifically the Big Five personality traits and intelligence. Findings suggest that the SBSOD scale has relatively high general factor saturation, and that a considerable portion of the variance in SBSOD scores is explained by other personality traits, including Conscientiousness $(r$ $=0.33)$, Intellect $(r=0.27)$, Emotional Stability $(r=0.26)$, and Extraver-

\footnotetext{
This research was supported in part by grant SMA-1419324 from the National Science Foundation to William Revelle and in part by the Spatial Intelligence and Learning Center (National Science Foundation Grant SBE0541957).

* Correspondence concerning this article should be addressed to David M. Condon, Department of Medical Social Sciences, Northwestern University, Chicago IL, 60611. Telephone number: (312) 503-7715. Email: david-condon@northwestern.edu.

${ }^{1}$ Northwestern University

${ }^{2}$ Case Western Reserve University

${ }^{3}$ University of California, Santa Barbara
} 
sion $(r=0.23)$. Cognitive ability was less highly correlated with SBSOD scores when measured at the level of general intelligence $(r=0.11)$ and in terms of mental rotation ability $(r=.07)$. Recommendations are given for revision of the SBSOD scale based on item-level analyses.

Keywords: Sense of direction, personality, Big Five, cognitive ability, individual differences.

\section{Introduction}

Each day that we venture into the world on foot or via wheeled vehicle, we travel over well-learned routes or enter unknown territory. Some of us approach new spatial environments with anxiety at the prospect of losing our way; others relish, and even seek, the experience of reaching a new destination aided by a map, verbal instructions, or merely a keen spatial awareness of the environment and the orientation of their body within it.

Sense of direction ("SOD") has previously been operationalized as the ability to locate and orient ourselves with respect to environmental space (Hegarty et al., 2002), and there is considerable evidence for the predictive utility of self-report measures of this psychological construct. In particular, prior research has demonstrated that self-reported Sense of Direction is positively correlated with proficiency at: (1) estimating distances $(r=0.00-$ 0.48, $n=24$ - 286; Hegarty et al., 2002, Ishikawa and Montello, 2006); (2) estimating direction under various conditions $(r=0.36-0.45, n=24-25$; Hegarty et al., 2002, Ishikawa and Montello, 2006, Montello and Pick, 1993); (3) giving, following, and remembering directions (see Hund and Padgitt, 2010); (4) maintaining an accurate orientation in complex environments $(r$ 
$=0.51-0.82, n=12-31$; Sholl, Kenny, and DellaPorta, 2006); and, perhaps most importantly, (5) wayfinding accuracy $(r(100)=0.20$; Hund and Padgitt, 2010; see also Kato and Takeuchi, 2003).

Given the scope of research evaluating the utility of SOD, relatively little research has investigated either the psychometric properties of the self-report scale by which it is most commonly measured - the Santa Barbara Sense of Direction scale ("SBSOD", Hegarty et al., 2002) - or the broader personality correlates. There are two noteworthy examples. Montello and Xiao (2011) evaluated the factor structure based on administration of the SBSOD to 5 small $(n=89-137)$ culture-specific samples. Bryant (1982) explored relationships between personality and SOD but employed methods that are now outdated due to advances in theory for both constructs. Sense of direction was assessed using a 50 item self-report measure that included items relating to styles of exploration, responses to disorientation, aesthetic ratings of the campus on which the data were collected, and self-estimates of spatial ability, direction-giving and direction-taking. Personality was assessed using the 18 scales of the California Psychological Inventory (Gough, 1975). None of the personality scales correlated significantly with self-reported SOD for males and females independently; only the Flexibility scale correlated significantly using the full sample $(r(64)=-.27)$.

The present study was conducted to first evaluate the factor structure of the SBSOD and then situate it within modern frameworks of individual differences (specifically the Big Five personality traits and intelligence). Analyses of the factor structure for the SBSOD were conducted in order to evaluate its general factor saturation; these analyses were motivated by the fact that 
SOD is typically conceptualized as a unitary construct (Montello and Xiao, 2011). To the extent that lower-order structure exists in the SBSOD, we endeavored to identify these facets and their relationship to personality. The second set of analyses - situating the SBSOD among prominent domains of individual differences - was conducted in order to explore the degree to which Sense of Direction reflects an ability rather than a temperamental orientation. Despite prior research on this topic (Hegarty et al., 2002, Hegarty, Montello, Richardson, Ishikawa, and Lovelace, 2006, Hund and Nazarczuk, 2009), it remains unclear whether SOD is merely a by-product of spatial and/or navigational ability or more likely to be a manifestation of "personality" as defined by an individual's stable pattern of affective, behavioral, and cognitive interaction with his or her environment (Ortony, Norman, and Revelle, 2005, Revelle, Wilt, and Condon, 2011).

It should be noted that the distinction between these two possibilities is spurious, as both temperament and cognitive abilities (as well as interests and other aspects of motivation among other sources of individual differences) are fundamental features of personality, writ large. Cognitive abilities and temperament differ from an assessment perspective in that the former typically measures maximal performance and the latter assesses typical behavior, but both are highly predictive domains of individual differences research. As such, a more precise description of the question at hand is whether Sense of Direction is more closely related to the cognitive abilities or temperamental variables; it is a "personality" characteristic in either case.

Based on research suggesting that various personality characteristics promote or hinder one's tendency to actively engage with the environment 
(Bryant, 1982), we hypothesized that individual differences in SOD would be more highly correlated with the dimensions of the Big Five (Goldberg, 1990) than cognitive ability. More specifically, the characteristics of extraversion (energy, enthusiasm, approach behavior), conscientiousness (attention to detail, organization, diligence), and openness (curiosity, ingenuity, adventurousness) were expected to show the strongest positive relationships on the grounds that these traits promote active engagement. Similarly, the avoidance processes associated with neuroticism (anxiety, withdrawal, and self-consciousness) were expected to be negatively related to SOD because they tend to hinder active engagement with the environment. No hypotheses were made about the relation between agreeableness (the tendency to be polite, cooperative, and trustful) and SOD. In the event that SOD was more closely related to ability than the Big Five traits, it was expected to be most highly associated with spatial ability.

\section{Method}

\subsection{Participants}

Participants were 12,155 individuals (64\% female) who completed an online survey at SAPA-project.org (previously test.personality-project.org) in exchange for customized feedback about their personalities. The data for this sample were collected over the course of two time periods; first from August 5, 2009 to August 21, 2009 and then from November 18, 2011 to January 11, 2012. All data were self-reported. The mean self-reported age was 25.5 years $(s d=10.6$, median $=21)$ with a range from 14 to 90 years. All educational attainment levels were well represented in the sample: $24 \%$ 
had 12 or fewer years of education; $20 \%$ had some graduate or professional school experience; the remainder had college degrees (35\%) or were currently enrolled (20\%). Approximately $30 \%$ of the sample $(n=3,585)$ provided their country of origin data. Of this subset, 105 countries were represented overall (73\% U.S.). Participants were also given the option to provide self-reported achievement test scores. Approximately $15.4 \%$ of participants provided selfreported scores for at least one of the tests. Mean combined scores for the SAT Critical Reading and Math were 1241 (median $=1260, s d=199)$. Mean ACT Composite scores were $25.9($ median $=26, s d=5)$.

\subsection{Measures}

Participants were administered items from three sets of scales using the Synthetic Aperture Personality Assessment ("SAPA") technique (Revelle, Wilt, and Rosenthal, 2010), a variant of matrix sampling procedures discussed by Lord (1955) (see also Condon and Revelle, 2014). This technique makes use of random sampling from large sets of personality and ability items in order to create synthetic correlations across a wide range of constructs even though a reasonably small subset of the items are presented to any one subject. Exclusive of questions regarding participant characteristics (i.e., gender, age, country of origin, education and achievement test scores), each participant was administered 72 to 76 items in total; variability in the total number was independent of participant characteristics.

The three sets of scales were the 15 item Santa Barbara Sense of Direction scale ("SBSOD", Hegarty et al., 2002, Table 2), the International Personality Item Pool 100-Item Set of Big-Five Factor Markers ("IPIP100", Goldberg, 1992, 1999), and 52 items from the International Cognitive Ability Resource 
("ICAR", Condon and Revelle, 2014). Each participant was administered 10 of the 15 SBSOD items at random, 50 of the IPIP100 items (10 items each for Conscientiousness, Agreeableness, Emotional Stability, Extraversion, and Intellect), and 12 to 16 of the 52 ICAR items assessing Three-Dimensional Rotation, Verbal Reasoning, Matrix Reasoning, and Letter and Number Series item types.

The use of SAPA administration procedures for structural analyses requires that the pairwise administration of items is high enough to provide stability in the covariance matrix (Kenny, 2012, Condon and Revelle, 2014). The number of pairwise administrations suggested more-than-adequate stability across all items $(m=1,702$; median $=1,904 ; s d=1,563)$ and for the SBSOD items independently $(m=1,926 ;$ median $=1,925 ; s d=30)$.

\subsection{Analyses}

Analyses were conducted in the R computing environment (R Core Team, 2014), primarily using the psych package (Revelle, 2014). Internal consistency measures and assessment of the omega hierarchical general factor saturation (Revelle, 2014, Revelle and Zinbarg, 2009, Zinbarg, Yovel, Revelle, and McDonald, 2006) were based on the Pearson correlations between items. The factoring method used for the omega analyses minimized the residuals of the Ordinary Least Squares ("OLS") (Revelle, 2014). The polychoric/tetrachoric correlations between items were used to score the scales and conduct analyses based on two-parameter Item Response Theory (Baker, 1985, Embretson, 1996, Revelle, 2014) as this allowed for estimation of the correlations between items as if they had been measured continuously (Revelle and Condon, in press). Note that the SAPA sampling procedure described above results in 
data which are "massively missing completely at random", and that the range of the confidence intervals for the correlations is dependent on the number of pairwise administrations between items. Probability values for the correlations were estimated based on bootstrapping procedures provided in the psych package (see 'cor.ci', Revelle, 2014).

\section{Results}

Internal consistencies for the IPIP100 scales and the ICAR scales are reported in Table 1. All of the IPIP scales and most of the ICAR scales demonstrated high consistency among the items. The only prominent exception were the Matrix Reasoning items $\left(\alpha=0.53, \omega_{h}=0.49, \omega_{t}=0.58\right)$. This likely reflects the fact that relatively few items of this type were administered.

Correlations between the items of the SBSOD and the scales for the IPIP100 and the ICAR60 are presented in Figure 1 (full text for the SBSOD items is listed in Table 2). The standard deviation of these correlations were generally quite low (0.01 to 0.03$)$ due the large number of pairwise administrations between items. The full distribution of the standard deviations between correlations is depicted in Figure 2. Most of the correlations between the SBSOD items and the other scales were small to moderate in magnitude; all but one ranged between -0.28 and 0.28 . The exception was SBSOD item 2 ("Have a poor memory for where I left things."), which had a high correlation (-0.45) with the Conscientiousness scale of the IPIP100. The SBSOD items were generally uncorrelated with the ICAR scales with two notable exceptions; item 7 ("Enjoy reading maps.") and item 9 ("Am very good at reading maps.") both had low, positive correlations with the ICAR scales ( $r$ 
$=0.15-0.25$ and $r=0.14-0.21$, respectively). None of the SBSOD items correlated significantly more with the spatially-oriented Three-Dimensional Rotation items than with the other cognitive ability item types. Among the correlations between SBSOD items, two items - item 2 and item 12 ("It's not important to me to know where I am.") - demonstrated notably smaller correlations with the SBSOD scale scores $(r=-0.38$ for item 2 and $r=-0.24$ for item 12) than the rest of the items in the set.

Internal consistency measures of the SBSOD indicated a high degree of general factor saturation, with an $\omega_{\text {hierarchical }}$ of 0.73 . Other common indicators of general factor saturation were uniformly high: Cronbach's $\alpha=0.88$, Guttman's $\lambda_{6}=0.89$, and McDonald's $\omega_{\text {total }}=0.90$. Loadings of each item on the general factor as well as the group factors that emerged from analyses of the general factor saturation are shown in Table 2. The correlations between the three secondary factors are shown in Table 3 . Three group factors were chosen in order to facilitate interpretation of the underlying structure of the items. In addition to high general factor saturation, the moderate to high correlations between secondary group factors also provide evidence for unidimensionality among the items.

Consistent with the raw correlations, items 2 and 12 were the only SBSOD items that did not load highly on the general factor as all other items had loadings of 0.38 or higher. The first group factor appears to be welldefined by items assessing the tendency to remember routes and maintain one's orientation. The second and third group factors were defined by items assessing enjoyment and skill related to reading maps and giving directions, respectively. Note that item 12 demonstrated consistently low loadings on 
all of the secondary factors as well as the general factor (0.14).

Results from the analyses based on Item Response Theory are given in Figure 3 and Table 4 . The information functions for each item are summarized for several levels of the latent trait in Table 4. All of these analyses presume unidimensionality in the underlying construct as supported by the high general factor saturation. The information function for the scale as a whole (plotted in Figure 3) reflects high reliability at low levels of the latent trait and lower (though still adequate) reliability at the higher end of the range.

\section{Discussion}

These findings suggest that scores on the Santa Barbara Sense of Direction scale demonstrate a clear general factor, and that a considerable portion of the variance in these scores is explained by other aspects of personality. They also suggest that revision of the SBSOD may be in order.

All of the Big Five traits except Agreeableness correlated with SBSOD scores in the hypothesized direction. In other words, SBSOD scores were positively correlated with Conscientiousness $(r=0.32, p<.001)$, Extraversion $(r=0.22, p<.001)$, Intellect $(r=0.26, p<.001)$, and Emotional Stability (low Neuroticism) $(r=0.26, p<.001)$. The correlations between SBSOD and the cognitive ability measures were notably lower than those of personality ( $r$ 's between .07 and 0.13 ). While the ICAR correlations with SBSOD were all similar in magnitude, it was notable that the smallest of these correlations involved the most spatially related item type - the Three-Dimensional Rotation items; this is consistent with prior findings of relatively low correla- 
tions between Sense of Direction and mental rotation (Bryant, 1982, Hegarty et al., 2002, 2006). When considered together, this combination of results suggests that Sense of Direction is less related to cognitive ability (including mental rotation ability) than to Big Five traits, and that the direction of these associations is consistent with active engagement with the environment. Although it is also possible that the positive correlations between the Big Five and SBSOD scores result from socially desirable responding, correlations were relatively lower between SBSOD and Agreeableness $(r=$ 0.07, $p<.001$ ), the Big Five scale which is most typically influenced by social-desirability response patterns.

SBSOD scores were also positively associated with several demographic characteristics, including gender (towards males), age, and higher educational attainment. The association with age, though not explicitly hypothesized, is consistent with the possibility that SOD is associated with active engagement in that an integral by-product of engagement is experience. In other words, both the degree and frequency of engagement with the environmental space should be expected to affect one's Sense of Direction. It is likely that the positive association between SBSOD and educational attainment is a function of the association with age as these two demographic variables are often highly correlated. The fact that SBSOD scores are higher for males is consistent with prior research (Bryant, 1982, Hegarty et al., 2006, Kozlowski and Bryant, 1977, Lawton, 1994, Prestopnik and Roskos-Ewoldsen, 2000, Sholl, Acacio, Makar, and Leon, 2000). Identifying the mechanism that underlies this association, however, remains an important topic for future research.

With regards to the item-level factor structure, SBSOD items assessing 
the propensity to actively engage with maps and directions form somewhat distinct - though highly related - sub-factors of the Sense of Direction construct. The two most highly-loaded items in the "Engagement with Maps" sub-factor (see Table 2) also had the highest loadings with the cognitive ability scales (see Figure 1). Further, all four of the items in this sub-factor demonstrated higher-than-average correlations with gender, age and education. The two items comprising the third sub-factor, "Engagement with Directions," both had high loadings on the general factor but were uncorrelated with the other sub-factors. These items suggest that one's attitude about giving directions is a reasonable proxy for Sense of Direction.

Factor analyses also provided strong support for the removal of two items from the SBSOD scale. Item 12 does not contribute meaningfully to either the general factor or any of the secondary factors. In the case of item 2 , the general factor loadings are marginal while its correlations with Conscientiousness are unusually high. While the 13 items remaining after removal of items 2 and 12 are likely to be few enough for most assessment purposes, further reduction could be achieved by using the item information characteristics from the IRT analyses. The IRT-based item parameters suggest that a subset of the most informative items listed in Table 4 would provide adequate assessment for Sense of Direction with little reduction in reliability across the range of the latent trait.

\section{Conclusion}

These data suggest that engagement (and resultant experience) with one's environment may play a role in shaping attitudes about Sense of Direction, 
and this in turn suggests the need for future research which explores the degree to which Sense of Direction is amenable to manipulation based on engagement behaviors that correlate with personality traits such as Conscientiousness. The results presented here clearly demonstrate that, while cognitive ability was positively correlated with Sense of Direction, larger correlations were evident between Sense of Direction and the Big Five and demographic characteristics, including age. 


\section{References}

Baker, F. B., Oct. 1985. The basics of item response theory. Heinemann Educational Books, Portsmouth, NH.

Bryant, K. J., 1982. Personality correlates of sense of direction and geographic orientation. Journal of Personality and Social Psychology 43 (6), 1318 1324.

Condon, D. M., Revelle, W., 2014. The International Cognitive Ability Resource: Development and initial validation of a public-domain measure. Intelligence 43, 52-64.

Embretson, S. E., 1996. The new rules of measurement. Psychological Assessment 8 (4), 341-349.

Goldberg, L. R., 1990. An alternative "description of personality": The BigFive factor structure. Journal of Personality and Social Psychology 59 (6), $1216-1229$.

Goldberg, L. R., 1992. The development of markers for the Big-Five factor structure. Psychological Assessment 4 (1), 26-42.

Goldberg, L. R., 1999. A broad-bandwidth, public-domain, personality inventory measuring the lower-level facets of several Five-Factor Models. In: Mervielde, I., Deary, I., De Fruyt, F., Ostendorf, F. (Eds.), Personality Psychology in Europe. Tilburg University Press, Tilburg, The Netherlands, pp. $1-7$. 
Gough, H. G., 1975. Manual for the California Psychological Inventory. Consulting Psychologists Press.

Hegarty, M., Montello, D. R., Richardson, A. E., Ishikawa, T., Lovelace, K., 2006. Spatial abilities at different scales: Individual differences in aptitudetest performance and spatial-layout learning. Intelligence 34 (2), 151-176.

Hegarty, M., Richardson, A. E., Montello, D. R., Lovelace, K., Subbiah, I., 2002. Development of a self-report measure of environmental spatial ability. Intelligence 30 (5), 425-447.

Hund, A. M., Nazarczuk, S. N., 2009. The effects of sense of direction and training experience on wayfinding efficiency. Journal of Environmental Psychology 29 (1), 151-159.

Hund, A. M., Padgitt, A. J., 2010. Direction giving and following in the service of wayfinding in a complex indoor environment. Journal of Environmental Psychology 30, 553-564.

Ishikawa, T., Montello, D. R., Mar. 2006. Spatial knowledge acquisition from direct experience in the environment: Individual differences in the development of metric knowledge and the integration of separately learned places. Cognitive Psychology 52 (2), 93-129.

Kato, Y., Takeuchi, Y., Jun. 2003. Individual differences in wayfinding strategies. Journal of Environmental Psychology 23 (2), 171-188.

Kenny, D. A., 2012. Measuring model fit. Retrieved November 7, 2012, from http://www.davidakenny.net/cm/fit.htm. 
Kozlowski, L. T., Bryant, K. J., 1977. Sense of direction, spatial orientation, and cognitive maps. Journal of Experimental Psychology: Human Perception and Performance 3 (4), 590-598.

Lawton, C. A., 1994. Gender differences in way-finding strategies: Relationship to spatial ability and spatial anxiety. Sex Roles 30 (11-12), 765-779.

Lord, F. M., 1955. Sampling fluctuations resulting from the sampling of test items. Psychometrika 20 (1), 1-22.

Montello, D. R., Pick, H. L., May 1993. Integrating knowledge of vertically aligned large-scale spaces. Environment and Behavior 25 (3), 457-484.

Montello, D. R., Xiao, D., 2011. Linguistic and cultural universality of the concept of sense-of-direction. In: Spatial Information Theory. Springer Berlin Heidelberg, pp. 264-282.

Ortony, A., Norman, D. A., Revelle, W., 2005. Affect and proto-affect in effective functioning. In: Fellous, J. M., Arbib, M. A. (Eds.), Who Needs Emotions? The Brain Meets the Machine. Oxford University Press, New York, pp. 173-202.

Prestopnik, J. L., Roskos-Ewoldsen, B., 2000. The relations among wayfinding strategy use, sense of direction, sex, familiarity, and wayfinding ability. Journal of Environmental Psychology 20, 177-191.

R Core Team, 2014. R: A Language and Environment for Statistical Computing. R Foundation for Statistical Computing, Vienna, Austria, ISBN 3-900051-07-0.

URL http://www.R-project.org/ 
Revelle, W., 2014. psych: Procedures for psychological, psychometric, and personality research. Northwestern University, Evanston, Illinois, r package version 1.4.8.

URL http://personality-project.org/r/psych.manual.pdf

Revelle, W., Condon, D. M., in press. Reliability. In: Irwing, P., Booth, T., Hughes, D. (Eds.), Wiley-Blackwell Handbook of Psychometric Testing. Wiley-Blackwell.

Revelle, W., Wilt, J., Condon, D. M., 2011. Individual differences and differential psychology: A brief history and prospect. In: Chamorro-Premuzic, T., von Stumm, S., Furnham, A. (Eds.), The Wiley-Blackwell Handbook of Individual Differences. Wiley-Blackwell, Oxford, Ch. 1, pp. 3-38.

Revelle, W., Wilt, J., Rosenthal, A., 2010. Individual differences in cognition: New methods for examining the personality-cognition link. In: Gruszka, A., Matthews, G., Szymura, B. (Eds.), Handbook of Individual Differences in Cognition: Attention, Memory and Executive Control. Springer, New York, Ch. 2, pp. 27-49.

Revelle, W., Zinbarg, R. E., Mar. 2009. Coefficients alpha, beta, omega, and the glb: Comments on Sijtsma. Psychometrika 74 (1), 145-154.

Sholl, J. M., Acacio, J. C., Makar, R. O., Leon, C., 2000. The relation of sex and sense of direction to spatial orientation in an unfamiliar environment. Journal of Environmental Psychology 20 (1), 17-28.

Sholl, M. J., Kenny, R. J., DellaPorta, K. A., 2006. Allocentric-heading recall 
and its relation to self-reported sense-of-direction. Journal of Experimental Psychology 32 (3), 516-533.

Zinbarg, R. E., Yovel, I., Revelle, W., McDonald, R. P., 2006. Estimating generalizability to a latent variable common to all of a scale's indicators: A comparison of estimators for omega hierarchical. Applied Psychological Measurement 30 (2), 121-144. 


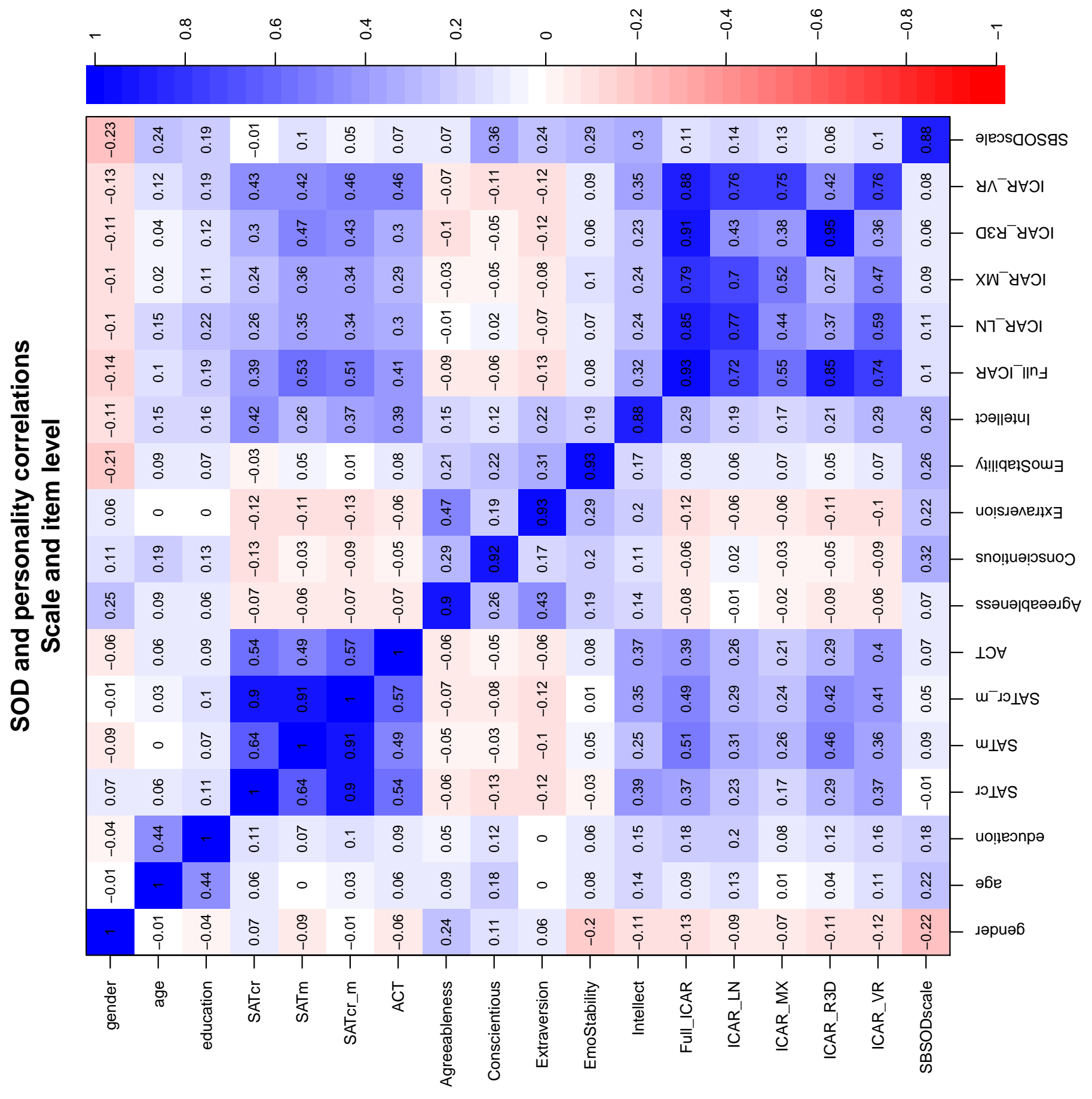

Figure 1: SOD and personality correlations on the scale and item level

Notes: Correlations have been corrected for item overlap where necessary. Correlations above the diagonal also reflect corrections for attenuation. The SBSODscale is based on all of the SOD items. The Full_ICAR score is comprised of the four ICAR subtests. SATcr is SAT - Critical Reading; SATm is SAT - Math; SATcr_m is SAT - Combined; ICAR_LN is Letter and Number Series; ICAR_MX is Matrix Reasoning; ICAR_R3D is Three-Dimensional Rotation; ICAR_VR is Verbal Reasoning. Gender is coded as 1 for males and 2 for females. 


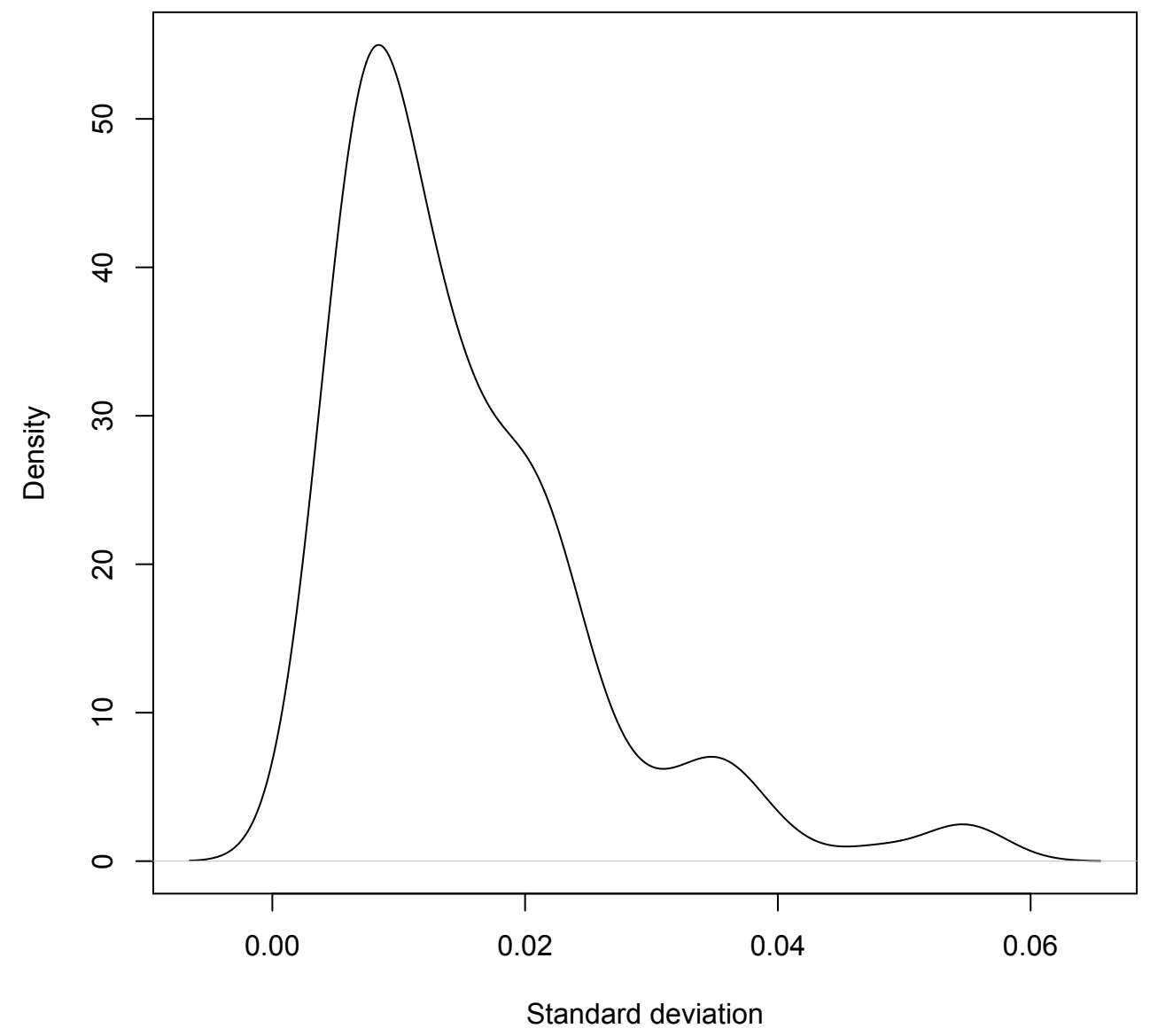

Figure 2: Distribution of the standard deviations of the correlations shown in Figure 1 


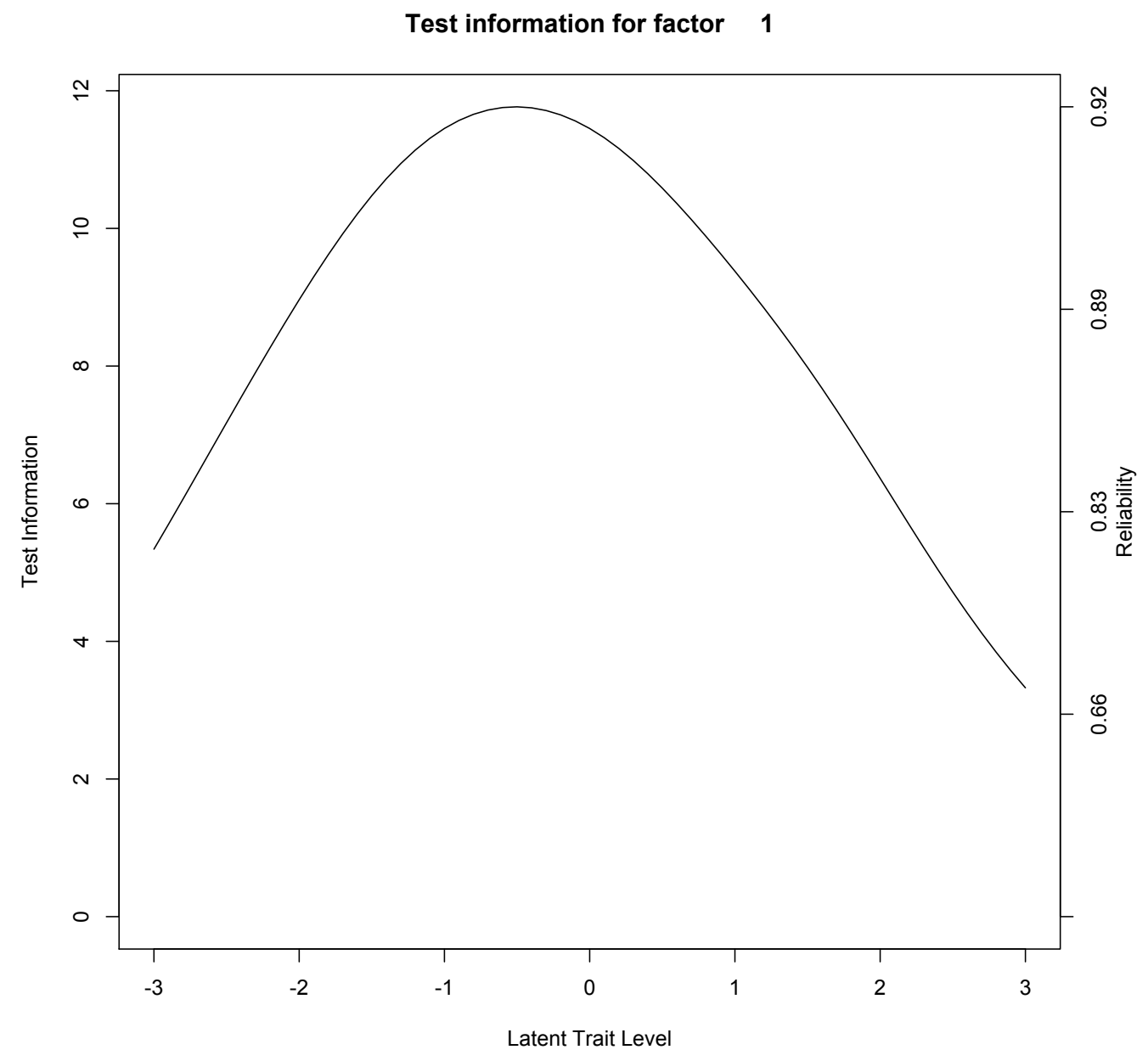

Figure 3: SBSOD test information function 
Table 1: Internal consistencies for the IPIP and ICAR scales

\begin{tabular}{llcccc}
\hline & $\alpha$ & $\omega_{h}$ & $\omega_{t}$ & items \\
\hline IPIP100 & & & & & \\
& Agreeableness & 0.91 & 0.80 & 0.92 & 20 \\
& Conscientiousness & 0.92 & 0.67 & 0.93 & 20 \\
Extraversion & 0.93 & 0.76 & 0.94 & 20 \\
Emotional Stability & 0.93 & 0.72 & 0.94 & 20 \\
& Intellect & 0.88 & 0.58 & 0.91 & 20
\end{tabular}

ICAR

\begin{tabular}{lcccc} 
Full ICAR & 0.96 & 0.60 & 0.97 & 52 \\
Letter Number Series & 0.77 & 0.71 & 0.82 & 9 \\
Matrix Reasoning & 0.53 & 0.49 & 0.58 & 4 \\
Three-Dimensional Rotation & 0.97 & 0.60 & 0.99 & 24 \\
Verbal Reasoning & 0.76 & 0.64 & 0.78 & 15 \\
\hline
\end{tabular}

Note: $\alpha=$ Cronbach's alpha, $\omega_{h}=$ omega hierarchical, $\omega_{t}=$ omega total 
Table 2: SBSOD items and their factor loadings on the general

factor and three oblique secondary factors

\begin{tabular}{|c|c|c|c|c|}
\hline \multirow[b]{2}{*}{ Items by Secondary Factor } & \multicolumn{4}{|l|}{ General } \\
\hline & Factor & Factor 1 & Factor 2 & Factor 3 \\
\hline \multicolumn{5}{|l|}{ Orientation } \\
\hline Item 14. Can usually remember a new route after I have traveled it only once. & 0.57 & 0.51 & -0.01 & -0.03 \\
\hline Item 10. Don't remember routes very well while riding as a passenger in a car. (R) & 0.54 & 0.51 & -0.06 & -0.02 \\
\hline Item 4 . Have a very good sense of direction. & 0.73 & 0.40 & 0.10 & 0.08 \\
\hline Item 6 . Very easily get lost in a new city. (R) & 0.58 & 0.35 & 0.12 & 0.03 \\
\hline Item 15. Don’t have a very good "mental map" of my environment". (R) & 0.47 & 0.30 & 0.05 & 0.03 \\
\hline Item 8. Have trouble understanding directions. $(\mathrm{R})$ & 0.58 & 0.23 & -0.01 & 0.16 \\
\hline Item 3. Am very good at judging distances. & 0.47 & 0.22 & 0.08 & 0.07 \\
\hline Item 2. Have a poor memory for where I left things. (R) & 0.26 & 0.16 & -0.05 & 0.05 \\
\hline \multicolumn{5}{|l|}{ Engagement with Maps } \\
\hline Item 7. Enjoy reading maps. & 0.51 & -0.03 & 0.73 & -0.02 \\
\hline Item 9. Am very good at reading maps. & 0.64 & 0.10 & 0.51 & 0.06 \\
\hline Item 5. Tend to think of my environment in terms of cardinal directions $(\mathrm{N}, \mathrm{S}, \mathrm{E}, \mathrm{W})$. & 0.38 & 0.10 & 0.26 & 0.03 \\
\hline Item 13. Usually let someone else do the navigational planning for trips. (R) & 0.60 & 0.15 & 0.20 & 0.12 \\
\hline \multicolumn{5}{|l|}{ Engagement with Directions } \\
\hline Item 1. Am very good at giving directions. & 0.80 & 0.00 & 0.00 & 0.40 \\
\hline Item 11. Don't enjoy giving directions. (R) & 0.61 & -0.02 & 0.00 & 0.32 \\
\hline Item 12. It's not important to me to know where I am. (R) & 0.14 & 0.06 & 0.05 & 0.05 \\
\hline
\end{tabular}

Note: (R) indicates that the item was reverse scored. 
Table 3: Correlations between the secondary factors of the SBSOD

Factor 1 Factor 2 Factor 3

\begin{tabular}{llll}
\hline Factor 1 & 1.00 & & \\
Factor 2 & 0.48 & 1.00 & \\
Factor 3 & 0.69 & 0.56 & 1.00 \\
\hline
\end{tabular}


Table 4: Item and test information for the SBSOD

\begin{tabular}{lccccccc}
\hline & \multicolumn{7}{c}{ Latent Trait Level } \\
& \multicolumn{7}{c}{ (normal scale) } \\
Item & -3 & -2 & -1 & 0 & 1 & 2 & 3 \\
\hline Item 14 & 0.43 & 0.77 & 1.01 & 0.99 & 0.76 & 0.46 & 0.22 \\
Item 10 & 0.30 & 0.59 & 0.91 & 1.02 & 0.85 & 0.53 & 0.26 \\
Item 4 & 1.04 & 1.71 & 1.86 & 1.54 & 1.15 & 0.84 & 0.26 \\
Item 6 & 0.32 & 0.64 & 0.95 & 1.06 & 0.91 & 0.61 & 0.31 \\
Item 15 & 0.40 & 0.54 & 0.61 & 0.56 & 0.43 & 0.29 & 0.17 \\
Item 8 & 0.53 & 0.77 & 0.87 & 0.78 & 0.57 & 0.34 & 0.17 \\
Item 3 & 0.28 & 0.41 & 0.51 & 0.53 & 0.47 & 0.36 & 0.24 \\
Item 2 & 0.11 & 0.12 & 0.13 & 0.13 & 0.13 & 0.12 & 0.10 \\
Item 7 & 0.23 & 0.37 & 0.52 & 0.61 & 0.58 & 0.45 & 0.30 \\
Item 9 & 0.44 & 0.81 & 1.09 & 1.09 & 0.85 & 0.51 & 0.23 \\
Item 5 & 0.16 & 0.22 & 0.30 & 0.35 & 0.36 & 0.32 & 0.26 \\
Item 13 & 0.30 & 0.57 & 0.83 & 0.94 & 0.81 & 0.53 & 0.28 \\
Item 1 & 0.51 & 0.98 & 1.28 & 1.23 & 0.97 & 0.62 & 0.27 \\
Item 11 & 0.30 & 0.46 & 0.59 & 0.63 & 0.55 & 0.40 & 0.25 \\
Item 12 & 0.04 & 0.04 & 0.04 & 0.04 & 0.04 & 0.04 & 0.04 \\
\hline TIF & 5.34 & 8.96 & 11.45 & 11.45 & 9.38 & 6.37 & 3.32 \\
SEM & 0.43 & 0.33 & 0.30 & 0.30 & 0.33 & 0.40 & 0.55 \\
Reliability & 0.81 & 0.89 & 0.91 & 0.91 & 0.89 & 0.84 & 0.70 \\
\hline
\end{tabular}

